\title{
The aptness of antagonistic protocol in intracytoplasmic sperm injection: Embryologic study
}

\begin{abstract}
Background and objective: Infertility and poor ovarian response are serious problems in our society; fortunately the in vitro fertilization and intracytoplasmic sperm injection technique dissolve many of these problems. This study aimed to assess the effect of an individualized $\mathrm{GnRH}$ antagonist regimen on intracytoplasmic sperm injection process especially the aptness of it in given mature reiterative oocytes, normal fertilization, and acceptably zygote cleavage which enhance pregnancy.

Methods: The population of the study consisted of 877 couples attending the in vitro fertilization-infertility center in the maternity teaching hospital and chose to undergo intracytoplasmic sperm injection after using of antagonist protocol for stimulation of women ovaries. The women ages ranged between 24 and 44 years. The collected data included a number of reiterative oocytes; fertilization, embryo grades and lastly estimates the pregnancy results

Results: A significant association $(P<0.001)$ was found in the numbers of reiterative oocytes between the age groups of the study. A significant association $(P=0.005)$ was also found concerning to quality of fertilization process. The study of the zygote cleavage and blastomeres formation followed the fertilization show variable grade of embryos and analyzed data of embryo grades in this study indicate presence of significant association $(P=0.003)$. The rate of pregnancy showed significant association between the groups of the study with the $<30$ years age group have chance of pregnancy higher than other groups.
\end{abstract}

Conclusion: The antagonist protocol of ovary stimulation according to the results of this study is a qualified protocol; besides it is rapid and can be reversed. This protocol can give effective results in the intracytoplasmic sperm injection process especially for young women, and enhance the pregnancy.

Keywords: GnRH antagonist; Intracytoplasmic sperm injection stimulation; IVF protocol.

\section{Introduction}

Infertility and the problem of poor ovarian response in our century now a day is markedly increased due to an increase in the percentage of delayed marriage and advanced maternal age. Although the infertility is a source of profound psychological distress for those patients who choose to undergo in vitro fertilization (IVF), however often suffer additional anxiety and concern. ${ }^{1-2}$ However, a large number of IVF/ intracytoplasmic sperm injection (ICSI) cycles performed every year throughout the world. That means a considerable number of patients are affected factors influencing the incidence of the ovarian hyperstimulation syndrome (OHSS) include age, polycystic ovary syndrome (PCOS), use of exogenous HCG to induce ovulation, luteal phase supplementation with HCG, and more importantly, the stimulation regimen. ${ }^{3}$ In ovarian stimulation protocols, because administering exogenous $\mathrm{FSH}$ achieves an adequate follicular response, however, growing follicles become increasingly sensitive to and ultimately depend on the presence of both $\mathrm{LH}$ and $\mathrm{FSH}$ for their

* Department of Basic Science, College of Dentistry, Hawler Medical University, Erbil, I raq. 
https:/ / doi.org/ 10.15218/ zjms.2019.004

normal development. ${ }^{4}$ Furthermore, clinical data show that $\mathrm{LH}$ is essential for oocyte maturation in the normal menstrual cycle. ${ }^{5}$ The gonadotropin-releasing hormone $(\mathrm{GnRH})$ agonist protocol used in vitro fertilization (IVF) were reported and published for the first time in the 1980s. The GnRH agonists function depend on suppress luteinizing hormone $(\mathrm{LH})$ and prevent premature $\mathrm{LH}$ surges allowing optimal timing of human chorionic gonadotropin (hCG) administration and ovum collection, which improved IVF outcomes. ${ }^{6} \mathrm{GnRH}$ agonist long protocols have been considered as the standard and the most commonly used protocol during the last two decades. The GnRH agonist long protocol starting in the mid-luteal phase of the preceding cycle, typically involve about three weeks of $\mathrm{GnRH}$ analog treatment per cycle. ${ }^{7}$ However, the controlled ovarian hyperstimulation $(\mathrm{COH})$ protocols for in vitro fertilization (IVF) and intracytoplasmic sperm injection (ICSI) are stressful, invasive and can be associated with adverse pregnancy outcome, including miscarriage and ectopic pregnancy. ${ }^{8}$ More recently the $\mathrm{GnRH}$ antagonist $\mathrm{s}$ become available, which act in contrast to the agonist protocol. The antagonist protocol block pituitary $\mathrm{GnRH}$ receptors by competitive binding resulting in immediate and direct suppress of gonadotropin. ${ }^{9-10}$ $\mathrm{GnRH}$ antagonists can, therefore, be administered just before the expected LH surge that is mean between days 5-7 of stimulation when the risk of premature $\mathrm{LH}$ surge is most probable and need to be administered only for a few days (about 5-6 days per cycle.,11,12 Because of increase using of antagonist protocol now a day in many of infertility and IVF center in Erbil city due to the conception that this type of stimulation protocol can give opportunity to control the endogenous LH surge more rapidly and conveniently in order to get of mature oocytes, success fertilization, and good grade of embryo which enhance pregnancy. This study tries to investigate the Qualify of antagonist's protocol in the ICSI process especially the part which concerning to early embryonic stage.

\section{Methods}

This study was conducted in IVF -Infertility center in the Maternity Teaching Hospital in Erbil city, Iraq during the period from January 2013 to January 2016. The data of the couples attended the center and undergone ICSI were obtained which included a number of reiterative oocytes; fertilization, embryo grades (cleavages of the zygote for 3 or 5 days after fertilization) and lastly estimate the pregnancy rate. In this study, only the women who used the antagonistic program of ova stimulation was chosen in order to find the aptitude of the antagonist protocol in the formation of mature and good quality of ova which fertilize and divide equally to enhance pregnancy. In order to get most real results, some cases were excluded from this study included the teratospermia, necrospermia, asthinospermia, azoospermia and frozen sperms of both cases ejaculated sperms and testicular sperms aspiration (TESA) to limits the failure ratio. The population of the study consisted of 877 couples. The women ages ranged between 24 and 44 years. The cases classified according to age, reiterative oocytes $(<10,10-15,>15$ oocytes), fertilization which was checked after 18 hours of sperm injection (ICSI) using the inverted microscope to check the presence of two pronuclei (PN). The Good fertilization represents efficient fertilization that means the presence of $2 \mathrm{PN}$. The Poor fertilization represents incomplete fertilization or rapid fertilization; in this case, only one PN was seen. The not fertilized case reported when no PN was seen. Also, the embryo grad assayed according to the checked of fertilized ova after 24 hours of ICSI. Embryos are graded in the following manner. Grade 1 characterized by even number, regularly shaped blastomeres, intact zona pellucida and less than $10 \%$ fragmentation. Grade 2 
characterized by uneven number, irregular shape blastomeres, intact zona no more than $10 \%$ of fragmentation. Grade 3 which was similar to grade 2 with no more than $50 \%$ fragmentation. Grade 4 differs from grade 3 because containing more than $50 \%$ fragmentation and the last one degenerates' embryo which was characterized by dark appearance and shrieked shape. Data were coded and analyzed using the statistical package for the social sciences (version 18). Categorical variables were presented as the percentage. Chi-square test of association was used to compare proportions. A $P$ value of $\leq 0.05$ was considered statistically significant.

\section{Results}

The study included 877 cases of couples attended the IVF center of maternity teaching hospital in different months of the last three years, and the participated women undergone the antagonist program for ovary stimulation and then ICSI process. The ages of participated women ranged between 24- 44 years and the highest percentage of participated women include the age less than 30 years by (36.2\%) followed by the age group ranged between 30-34 years old with (34.9\%) whereas the low number of participating represent the older women who have age more than 40 years old with (7.1\%) as shown in Table 1 . The effect of antagonist's protocol on the reiterative oocytes, fertilization, grades of embryo and pregnancy rate is shown in Table 2 . This stimulation program can give the highest percentage $(51.7 \%)$ for the group of less than ten reiterative oocytes in contrast to the group which gives more than 15 ova (15.4\%). Also, according to data, we can see the high fertilization percentage $(87.8 \%)$ for this type of stimulation.

Table 1: Distribution of women by age.

\begin{tabular}{lcc}
\hline Age (Years) & No. & $\%$ \\
\hline$<30$ & 318 & 36.2 \\
$30-34$ & 306 & 34.9 \\
$35-39$ & 191 & 21.8 \\
$\geq 40$ & 62 & 7.1 \\
Total & 877 & 100 \\
\hline
\end{tabular}

Table 2: study data of reiterative oocytes, fertilization, embryo grads, and pregnancy ration.

\begin{tabular}{|c|c|c|c|}
\hline Variable & Group & No & $\%$ \\
\hline \multirow[t]{3}{*}{ Reiterative oocytes } & $<10$ & 453 & 51.7 \\
\hline & $10-15$ & 289 & 33 \\
\hline & $>15$ & 135 & 15.4 \\
\hline \multirow[t]{3}{*}{ Fertilization } & Not Fertilized & 39 & 4.4 \\
\hline & Good Fertilization & 770 & 87.8 \\
\hline & Poor Fertilization & 68 & 7.8 \\
\hline \multirow[t]{5}{*}{ Embryo grades } & Grade1 & 39 & 4.4 \\
\hline & Grade2 & 128 & 14.6 \\
\hline & Grade 3 & 139 & 15.8 \\
\hline & Grade 4 & 201 & 22.9 \\
\hline & degenerated & 370 & 42.2 \\
\hline \multirow[t]{2}{*}{ Pregnancy } & Negative & 493 & 56.2 \\
\hline & Positive & 384 & 43.8 \\
\hline Total & & 877 & 100 \\
\hline
\end{tabular}


Regarding the response of the different age groups to the antagonist protocol of stimulation, a very high significant association $(P<0.001)$ was found in the reiterative oocytes. The group of women less than 30 year gives a higher percentage $(22.9 \%)$ of reiterative Oocytes, and highly significant association $(P=0.005)$ of fertilization also found in this age group (89.3\%). A highly significant association $(P=0.003)$ in grades of the embryo was found between the different age groups (Table 3) which depend on the zygote cleavage, appearance of blastomeres and amount of fragmentation insides, as it explained in the methods of the study (Figures 1, 2, 3 and 4). The study found a very high significant association $(P<0.001)$ in the pregnancy rate as it is illustrated in Table 3 and Figure 5 between the ages groups, the younger women had more chance to get pregnant than other ages (51.9\%).

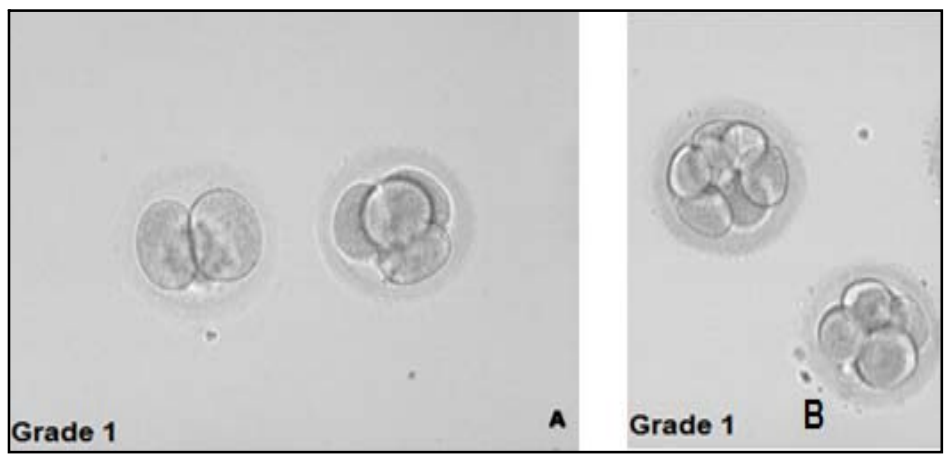

Figure 1: A \& B represent grade 1of embryo (cleavage of blastomeres in the $2^{\text {nd }}$ and third day of fertilization successional).

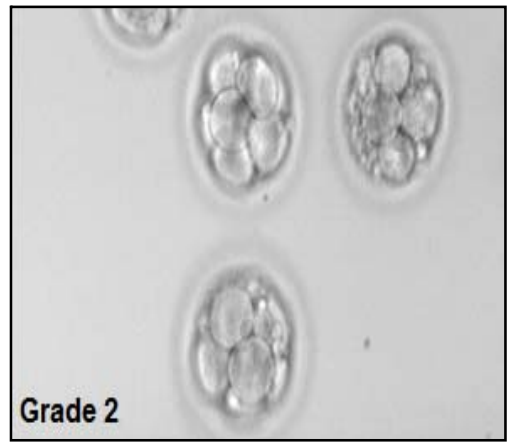

Figure 2: Represent grade 2 of the embryo.

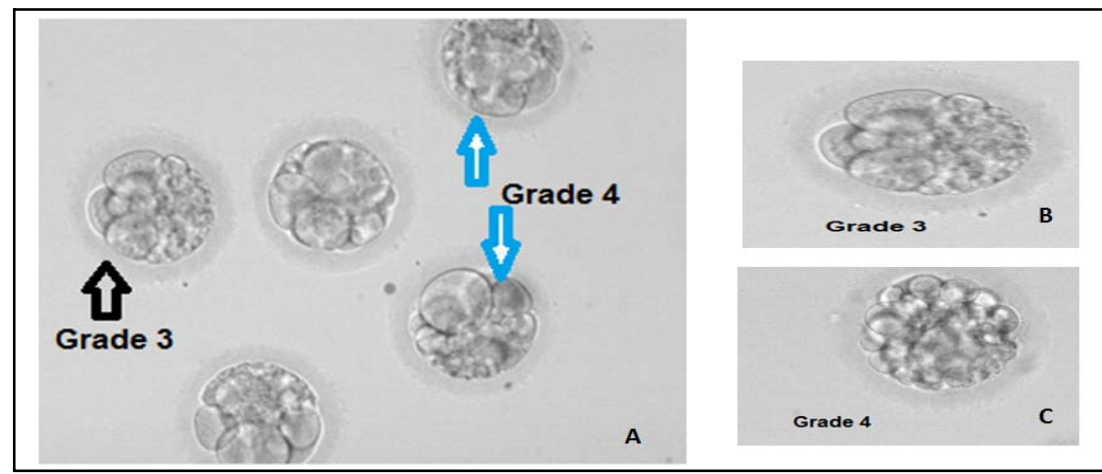

Figure 3: A- Represent grade 3 and 4 of embryo, B- Represent grade 3 and C- Represent grade 4. 
Table 3: Data of reiterative oocytes, fertilization, embryo grades and pregnancy ration in different age groups.

\begin{tabular}{|c|c|c|c|c|c|c|c|c|c|c|}
\hline \multirow{3}{*}{ Variable } & & \multicolumn{8}{|c|}{ Age groups } & \multirow{3}{*}{$P$ value } \\
\hline & & \multicolumn{2}{|c|}{$<30$} & \multicolumn{2}{|c|}{$30-34$} & \multicolumn{2}{|c|}{$35-39$} & \multicolumn{2}{|c|}{$\geq 40$} & \\
\hline & & No & $\%$ & No & $\%$ & No & $\%$ & No & $\%$ & \\
\hline \multirow{3}{*}{$\begin{array}{l}\text { Reiterative } \\
\text { oocytes }\end{array}$} & $<10$ & 106 & 33.3 & 171 & 55.8 & 123 & 64.4 & 53 & 85.5 & $<0.001$ \\
\hline & $10-15$ & 139 & 43.7 & 92 & 30.1 & 50 & 26.2 & 8 & 12.9 & \\
\hline & $>15$ & 73 & 22.9 & 43 & 14.1 & 18 & 9.4 & 1 & 1.6 & \\
\hline \multirow[t]{3}{*}{ Fertilization } & Not fertilized & 10 & 3.1 & 8 & 2.6 & 13 & 6.8 & 8 & 12.9 & 0.005 \\
\hline & Good fertilized & 284 & 89.3 & 273 & 89.2 & 161 & 84.3 & 52 & 83.9 & \\
\hline & Poor fertilized & 24 & 7.6 & 25 & 8.2 & 17 & 8.9 & 2 & 3.2 & \\
\hline \multirow{5}{*}{$\begin{array}{l}\text { Grade of } \\
\text { embryos }\end{array}$} & Grade 1 & 10 & 3.1 & 8 & 2.6 & 13 & 6.8 & 8 & 12.9 & 0.003 \\
\hline & Grade 2 & 45 & 14.1 & 49 & 16.0 & 26 & 13.6 & 8 & 12.9 & \\
\hline & Grade 3 & 45 & 14.1 & 48 & 15.7 & 29 & 15.2 & 16 & 25.8 & \\
\hline & Grade 4 & 67 & 21.1 & 72 & 23.5 & 53 & 27.7 & 9 & 14.5 & \\
\hline & Degenerated & 151 & 47.5 & 129 & 42.2 & 70 & 36.6 & 21 & 33.9 & \\
\hline \multirow[t]{2}{*}{ Pregnancy } & Negative & 153 & 48.1 & 166 & 54.2 & 132 & 69.1 & 42 & 67.7 & $<0.001$ \\
\hline & Positive & 165 & 51.9 & 140 & 45.8 & 59 & 30.9 & 20 & 32.3 & \\
\hline
\end{tabular}

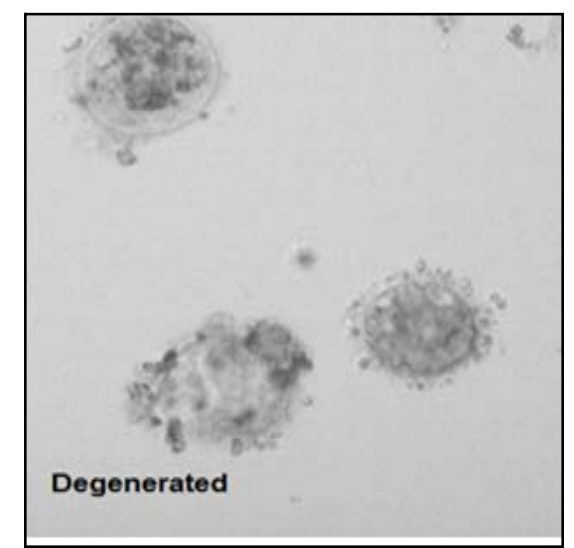

Figure 4: Represent degenerated embryo

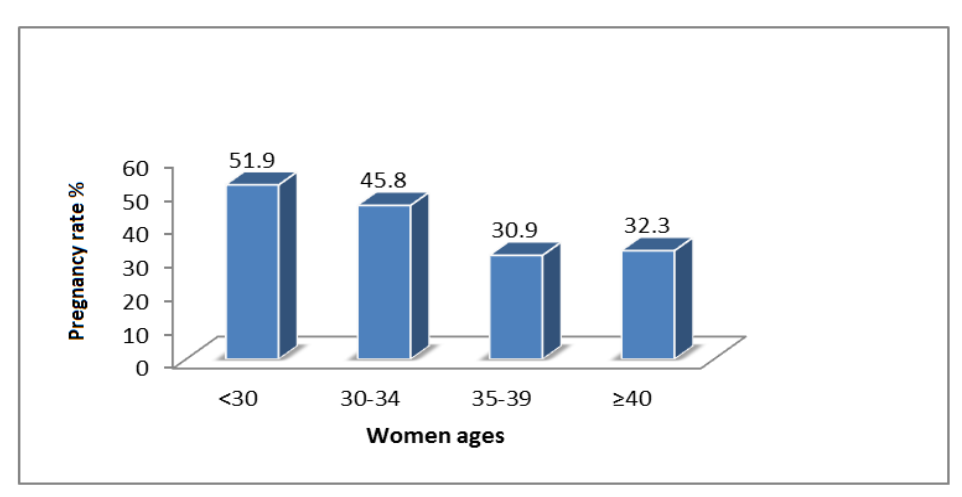

Figure 5: Pregnancy rate in different age group. 


\section{Discussion}

The antagonistic program of ovary stimulation is commonly used recently in many of infertility and IVF centers. GnRH antagonists competitively block pituitary $\mathrm{GnRH}$ receptors; including a rapid, reversible suppression of gonadotropin secretion. ${ }^{13}$ Pharmacological modes of $\mathrm{GnRH}$ antagonist's action can be administrated at mid-cycle to prevent a premature $\mathrm{LH}$ surge while not causing any suppression in the early follicular phase, which is a crucial time for follicular recruitment. The results of this study show qualified effect of this type of ovary stimulation protocols in the number of Reiterative oocytes (Table 2) when the reiterative oocytes mostly less than 10 . Therefore, this type of stimulation can be considered safer than other types. On the other hand, this stimulation program can be controlled by reversing its effect through the administration of $\mathrm{GnRH}$ agonist as it is reported by many studies ${ }^{14-16}$. The lower number of retrieved oocytes and subsequently lower estradiol levels might have a beneficial effect on the occurrence of ovarian hyperstimulation. Concerning to the effect of the antagonist on quality of retrieved oocytes; the results of fertilization and grades of the embryo which depend on the cleavages of zygote after fertilization that follow the ICSI process shows significant differences between the study groups as shown in Table 3 . The younger aged of the women (age $<30$, and 30-35) give a higher percentage of fertilization $(89.3 \%, 89.2 \%)$ and a higher percentage of grade 1, grade 2 embryos which considered as a perfect embryo to be transferred to the women uterus than the other groups. This agrees with other studies which suggested that the antagonist administration which is flexible regimen would lead to better clinical results and improve clinical outcome. ${ }^{17-18}$ Also, the study shows very high significant association $(P<0.001)$ between the study groups in the ratio of pregnancy (Figure 5). Although the younger ages ( $<30$ years) have the chance of pregnancy higher than other age the other ages also give a good percentage of outcome (positive percentage of pregnancy) if we compare the results of this study with expected results of IVF/ICSI. Also, that agrees with the conception which believed that antagonist protocol is acceptable to program mainly for the patient with unfavorable prognoses (e.g., older patient) or those whom previous cycles have been unsuccessful. ${ }^{19}$ A wide variety of $\mathrm{GnRH}$ antagonist protocols have been proposed, reflecting the fact that the protocol is still undergoing refinement. ${ }^{20-21}$

\section{Conclusion}

According to the results of this study, the antagonist program of ovary stimulation is a qualified or aptness protocol for IVF/ICSI process due to its results and its mode of action which was rapid and reversible. This protocol considered a safer program to use.

\section{Competing interests}

The author declares no competing interests.

\section{References}

1. Al-Inany HG, Abou-Setta AM, Aboulghar M. Gonadotrophin-releasing hormone antagonists for assisted conception. Cochrane Database Syst Rev 2006; 3:CD001750.

2. Cousineau TM, Domar AD. Psychological impact of infertility. Best Pract Res Clin Obstet Gynaecol 2007; 21:293-308.

3. Ragni G, Vegetti W, Riccaboni1 A, Engl B, Brigante C, Crosignani PG. Comparison of GnRH agonists and antagonists in assisted reproduction cycles of patients at high risk of ovarian hyperstimulation syndrome. Hum Reprod 2005; 20:2421-5.

4. Mochtar MH, Van der Veen F, Ziech M, van Wely M. Recombinant luteinizing hormone (rLH) for controlled ovarian hyperstimulation in assisted reproductive cycles. Cochrane Database Syst Rev 2007; 2:CD005070.

5. Hillier SG. Gonadotropic control of ovarian follicular growth and development. Mol Cell Endocrinol 2001; 179:39-46.

6. Lin $\mathrm{H}, \mathrm{Yu} \mathrm{L}$, Li L, Wang W, Dongzi Y, Zhang Q. Is a GnRH Antagonist Protocol Better in PCOS Patients? A Meta-Analysis of RCTs. PLOS ONE 2014; 9(3):e91796. 
7. Aygun BK, Kahraman S. Comparison of $\mathrm{GnRH}$ Agonist Long and Antagonist Protocols in the Same Normoresponder Patient Undergoing Assisted Reproductive Treatment. Fırat Tıp Dergisi 2010; 15(3):123-7.

8. Ioannidis G, Sacks G, Reddy N, Seyani L, Margara R, Lavery S, et al. Day 14 maternal serum progesterone levels predict pregnancy outcome in IVF/ICSI treatment cycles: a prospective study. Hum Reprod 2005; 20(3):741-6.

9. Fischl F, Nicollet B, Beher HM, Van der Ven $\mathrm{H}$, Simon A, Kilani $Z$, et al. The European and Middle East orgalturan, study group. Comparable clinical outcome using the $\mathrm{GnRH}$ antagonist ganirelix or a long protocol of the $\mathrm{GnRH}$ agonist triptorelin for the prevention of premature $\mathrm{LH}$ surges in women undergoing ovarian stimulation. Hum Reprod 2001; 16;644-51.

10. Mochtar $\mathrm{MH}$. The effect of an individualized $\mathrm{GnRH}$ antagonist protocol on folliculogenesis in IVF/ICSI. Hum Reprod 2004; 19(8):1713-8.

11. Borm G, Mannaerts B. Treatment with the gonadotropin-releasing hormone antagonist ganirelix in women undergoing controlled ovarian hyperstimulation with recombinant follicle stimulating hormone is effective, safe and convenient: results of controlled, randomized, multicentre trial. The European Orgalutran Study Group. Hum Reprod 2000; 15:1490-8.

12. Al-Inany HG, Abou-Setta AM, Aboulghar M. Gonadotrophin-releasing hormone antagonists for assisted conception: a Cochrane review. Reprod Biomed Online 2007; 14:640-9.

13. Diedrich K, Diedrich C, Santos E, Zoll C, al-Hasani S, Reissmann T, et al. Suppression of the endogenous LH-surge by the LH-RH antagonist Cetrorelix during ovarian stimulation. Hum Reprod 1994; 9:788-91.

14. Itskovitz-Eldor J, Kol S, Mannaerts B. Use of a single bolus of $\mathrm{GnRH}$ agonist triptorelin to trigger ovulation after $\mathrm{GnRH}$ antagonist ganirelix treatment in women undergoing ovarian stimulation for assisted reproduction, with special reference to the prevention of ovarian hyperstimulation syndrome: preliminary report: short communication. Hum Reprod 2000; 15:1965 -8 .

15. Fauser BC, de Jong D, Olivennes F, Wramsby $H$, Tay C, Itskovitz-Eldor J, et al. Endocrine profiles after triggering of final oocyte maturation with $\mathrm{GnRH}$ agonist after cotreatment with the $\mathrm{GnRH}$ antagonist ganirelix during ovarian hyperstimulation for in vitro fertilization. J Clin Endocrinol Metab 2002; 87:709-15.

16. Olivennes F, Fanchin R, Bouchard P, Taieb J, Frydman R. Triggering of ovulation by a gonadotropin-releasing hormone $(\mathrm{GnRH})$ agonist in patients pretreated with a $\mathrm{GnRH}$ antagonist. Fertil Steril 1996; 66:151-3.
17. Ludwig M, Katalinic A, Banz C, Schroder AK, Loning $\mathrm{M}$, Weiss JM, et al. Tailoring the $\mathrm{GnRH}$ antagonist cetrorelix acetate to individual patients' needs in ovarian stimulation for IVF: results of a prospective randomized study. Hum Reprod 2002; 17:2842-5.

18. Beckers NG, Macklon NS, Eijkemans MJ, Ludwig M, Felberbaum RE, Diedrich $\mathrm{K}$, et al. Nonsupplemented luteal phase characteristics after the administration of recombinant human chorionic gonadotropin, recombinant luteinizing hormone, or gonadotropin-releasing hormone $(\mathrm{GnRH})$ agonist to induce final oocyte maturation in in vitro fertilization patients after ovarian stimulation with recombinant follicle-stimulating hormone and $\mathrm{GnRH}$ antagonist cotreatment. J Clin Endocrinol Metab 2003; 88:4186-92.

19. Griesinger G, Felberbaum R, Diedrich K. GnRH antagonists in ovarian stimulation: a treatment regimen of clinicians' second choice? Data from the German national IVF registry. Hum Reprod 2005; 20:2373-5.

20. Macklon NS, Stouffer RL, Giudice LC, Fauser BC. The science behind 25 years of ovarian stimulation for in vitro fertilization. Endocr Rev 2006; 27:170-207.

21. Huirne JA, Homburg R, Lambalk CB. Are GnRH antagonists comparable to agonists for use in IVF? Hum Reprod 2007; 22:2805-13. 